\title{
180-kD Bullous Pemphigoid Antigen (BP180) Is Deficient in Generalized Atrophic Benign Epidermolysis Bullosa
}

\author{
Marcel F. Jonkman, * Marcelus C.J.M. de Jong, * Klaas Heeres, * Hendri H. Pas, * Jan B. van der Meer, * \\ Katsushi Owaribe, ${ }^{\ddagger}$ Ana M. Martinez de Velasco, ${ }^{\S}$ Carien M. Niessen, ${ }^{\S}$ and Arnoud Sonnenberg $\$$ \\ ${ }^{*}$ Department of Dermatology, University Hospital, Oostersingel 59, NL-9713 EZ Groningen, The Netherlands; ${ }^{\ddagger}$ Department of \\ Molecular Biology, Nagoya University School of Medicine, Furocho Chikusa-Ku, Nagoya-Shi 464-01, Japan; and ${ }^{\S}$ Department of Cell \\ Biology, The Netherlands Cancer Institute, Antoni van Leeuwenhoek Huis, Plesmanlaan 121, NL-1066 CX Amsterdam, The Netherlands
}

\begin{abstract}
Generalized atrophic benign epidermolysis bullosa (GABEB ) is a form of nonlethal junctional epidermolysis bullosa characterized by universal alopecia and atrophy of the skin. We report a deficiency of the $180-\mathrm{kD}$ bullous pemphigoid antigen in three patients with GABEB from unrelated families. We screened specimens of clinically normal skin from nine junctional epidermolysis bullosa patients ( 3 GABEB, 4 lethal, 1 cicatricial, 1 pretibial) by immunofluorescence using monoclonal antibodies to the $180-\mathrm{kD}$ and $230-\mathrm{kD}$ bullous pemphigoid antigens (BP180 and BP230). In the skin of the three GABEB patients there was no reactivity with antibodies to BP180, whereas staining for BP230 was normal. In the skin of the other six, non-GABEB patients, included in this study the expression of BP180 and BP230 was normal. Immunoblot analysis of cultured keratinocytes from one of the GABEB patients also failed to detect BP180 antigen, whereas BP230 was present in normal amounts. The deficient expression of BP180 is reflected in the RNA message, as in Northern blot analysis a reduced amount of BP180 transcripts, although of normal length, were detected. Interestingly, in another GABEB patient there were not-involved areas of skin, in which blistering could not be induced by rubbing. Biopsy material from these areas showed interrupted staining for BP180. There was no staining for BP180 in areas of clinically normal but involved skin of this patient.

In conclusion, this study reveals that the BP180 antigen is deficient and the BP180 mRNA is reduced in generalized atrophic benign epidermolysis bullosa. (J. Clin. Invest. 1995. 95:1345-1352.) Key words: junctional epidermolysis bullosa basement membrane $\cdot$ adhesion molecule $\bullet$ type XVII collagen • immunofluorescence
\end{abstract}

Address correspondence to Dr. Marcel F. Jonkman, Department of Dermatology, University Hospital, Oostersingel 59, NL-9713 EZ Groningen, The Netherlands. Tel: 31-50-619111; FAX: 31-50-612624.

Received for publication 20 May 1994 and in revised form $28 \mathrm{No}$ vember 1994.

1. Abbreviations used in this paper: BP180,180-kD bullous pemphigoid antigen; BP230, 230-kD bullous pemphigoid antigen; BP, bullous pemphigoid; EB, epidermolysis bullosa; EBMZ, epidermal basement membrane zone; GABEB, generalized atrophic benign epidermolysis bullosa; JEB, junctional epidermolysis bullosa.

J. Clin. Invest.

(c) The American Society for Clinical Investigation, Inc.

0021-9738/95/03/1345/08 \$2.00

Volume 95, March 1995, 1345-1352

\section{Introduction}

The $180-\mathrm{kD}$ bullous pemphigoid antigen $(\mathrm{BP} 180)^{1}$ is a putative cell-matrix adhesion molecule and is restricted to hemidesmosomes of stratified squamous epithelia. It is possibly involved in junctional epidermolysis bullosa (JEB), in which hemidesmosomes have been found to be abnormal (1). Unlike the 230$\mathrm{kD}$ bullous pemphigoid antigen (BP230), BP180 appeared to have both intracellular and extracellular domains $(2,3)$, which is in agreement with the deduced amino acid sequence of the cloned BP180 cDNA (4). Because BP180 is a transmembrane glycoprotein (3), it might be involved in those forms of JEB, in which, until now, no molecular abnormality has been revealed. In contrast, BP230 is localized entirely intracellularly (2) and is thus less likely to be involved in JEB, in which the split level occurs in the lamina lucida. Stanley and co-workers found no gross abnormalities of the BP230 gene in patients with lethal or non-lethal JEB (5). Molecular genetic studies of BP180 in JEB patients have not yet been performed.

In previous studies on the expression of the bullous pemphigoid antigen in JEB, sera from bullous pemphigoid (BP) patients were used, the specificity of which had not been analyzed by immunoblotting (6-9). The expression was found to be normal or variable. Later it became apparent that BP-serum is a mixture of polyclonal antibodies, which are very difficult to characterize, even after affinity-purification (10). Fortunately, reliable monospecific antibodies for each of the two major bullous pemphigoid antigens have now become available $(11,12)$.

In this study we used monoclonal antibodies specific for BP180 and BP230 $(11,12)$. In addition we studied the expression of the newly described $500-\mathrm{kD}$ hemidesmosomal plaque protein HD1 (13) in our series of JEB patients. We found a deficiency of the BP180 protein in patients with generalized atrophic benign epidermolysis bullosa (GABEB). The Northern blot analysis of cultured keratinocytes of one patient showed a reduced amount of BP180 mRNA of normal size. BP230 and HD1 were normally present in the GABEB patients.

Generalized JEB mitis is acknowledged by an international consensus committee to be a distinct form of JEB (14), differing from Herlitz disease (generalized JEB gravis) by its absence of anemia and growth retardation. The category generalized JEB mitis has not yet been split into subtypes and is also referred to as generalized atrophic benign epidermolysis bullosa (GABEB ) (15), epidermolysis bullosa atrophicans generalisata mitis (16) and nonlethal JEB (17) or non-Herlitz JEB. The first kindred with GABEB was described in 1976 by Hashimoto, Schnyder and Anton-Lamprecht and named Disentis type (McKusick \#22665), to the place of birth of the patients (18). The term GABEB and the clinical entity: nonlethal JEB with atrophic skin and alopecia, was introduced in 1982 by Hintner 
Table I. Summary of the Examined Antibodies to BP180, BP230, and HD1

\begin{tabular}{|c|c|c|c|c|c|c|}
\hline Antigen & Antibody & Gift from, reference & Antigen source & Antibody (iso)type & Antibody source & Titer used \\
\hline BP180 ec* & 1D1 & Ref. 3 & Bovine cornea & mo IgG1 & Mouse & 50 \\
\hline BP180 ic $^{\ddagger}$ & $1 \mathrm{~A} 8 \mathrm{c}$ & Ref. 3 & Bovine cornea & mo IgG1 & Mouse & 30 \\
\hline BP180 ec & R306.3 & Giudice, 20 & Recombinant BP180 & po IgG & Rabbit & 50 \\
\hline BP180 & S2204 & This study & Human skin & po serum & Human & 80 \\
\hline BP230 & $\mathrm{R} 815$ & Ref. 11 & Rat keratinocytes & mo IgGl & Mouse & 50 \\
\hline BP230 & $5 \mathrm{E}$ & Hashimoto, 12 & Human skin & mo IgG2 & Human & 50 \\
\hline BP230 & 10D & Hashimoto, 12 & Human skin & mo IgG2 & Human & 50 \\
\hline BP230/180 & S3105 & This study & Human skin & po serum & Human & 80 \\
\hline HD1 & HD121 & Ref. 13 & Bovine cornea & mo IgG1 & Mouse & 50 \\
\hline
\end{tabular}

$* e c$, extracellular domain; ${ }^{\ddagger} i c$, intracellular domain.

and Wolf (15). The clinical features of GABEB are continuous blistering since birth, cigarette paper-like atrophic depigmented skin at sites of recurrent blistering (no scarring or milia), normal growth and lack of anemia, moderate improvement during aging, dystrophic nails and dentition, mild mucous membrane involvement, and according to Hintner and Wolf (15), a typical pattern baldness with significant scalp atrophy, partial absence of eyelashes and eyebrows, and absence of pubic and axillary hair.

\section{Methods}

Patients. Three JEB cases with clinical GABEB phenotype from, according to the patients, unrelated families were included: a 40 -yr-old male (No. 1), product of a consanguineous marriage, a 42-yr-old female (No. 2) and a 27-yr-old female (No. 3) both products of nonconsanguineous marriages. All three patients had affected siblings. Patient 1 had undergone allogeneic kidney transplantation because of renal failure of unknown origin.

Six other JEB patients were examined: three patients with GB3negative lethal JEB (No. 4-6), one patient with GB3-positive lethal JEB (No. 7), one patient with cicatricial JEB (No. 8) (19), and one patient with localized pretibial JEB (No. 9). In addition we examined six patients with epidermolysis bullosa simplex (4 generalized and 2 localized EBS) to control for a possible aspecific variability of bullous pemphigoid antigen expression (6). Diagnosis was established in each patient on the basis of clinical findings, family history, immunofluorescence antigen mapping, and electron microscopy. Patients 4 and 5 with GB3-negative lethal JEB showed cytoplasmic redistribution of the $\alpha 6 \beta 4$ integrin in the absence of laminin-5, which we have published in a previous report (27).

Skin specimens. 4-mm punch biopsies were obtained from clinically normal skin of the flexor aspect of the upper arm of the patients. In the GABEB patients multiple skin biopsies were taken from various other parts of the body. We also obtained biopsies of clinically normal skin after inducing subclinical blistering by gently rubbing with a thumb. Skin specimens from healthy adults served as control tissues. The skin specimens were snap frozen for immunofluorescence or fixed with $2 \%$ glutaraldehyde for electron microscopy.

Source of antibodies. Characteristics of the antibodies used are summarized in Table I. Monoclonal antibodies 1D1, 1A8c, and HD121, raised against the hemidesmosome-enriched fraction of the Bowman's membrane of bovine corneae, and R815 against rat keratinocytes have been described $(3,11,13)$. The affinity-purified rabbit serum R306.3 ( =RNDla) raised against a recombinant extracellular domain of human BP180 (coding nucleotides 1624-1995 and 2593-2670) was a generous gift of Dr. G.J. Giudice (20). The specificity of antibodies was established by immunoblot on extracts of normal human keratinocytes (this study: 1A8c) and of the human carcinoma cell line A431 $(3,11)$. Monoclonal antibodies 5E and 10D, derived from an immortalized B cell line from a bullous pemphigoid patient, were a gift of Dr. Hashimoto (12). Monoclonal antibody GB3 against laminin-5 (nicein, kalinin, epiligrin) was provided through the courtesy of Dr. J.-P. Ortonne (21) and LH7:2 against collagen type VII was a gift of Dr. I. Leigh (22). In addition we used sera from bullous pemphigoid patients that recognizes both the 180- and 230-kD bands in the immunoblot (S3105, titer 1:640) ( see Fig. 6, lane $a$ ) or only the $180-\mathrm{kD}$ antigen (S2204). The $\alpha 6$ integrin subunit was stained with our moabs $\mathrm{GoH} 3(23)$ or $\mathrm{J} 8 \mathrm{H}(24)$ and the $\beta 4$ integrin subunit with moabs $439-9 \mathrm{~B}$ or $4.3 \mathrm{E} 1$, gifts of Dr. S.J. Kennell (25) and Dr. E. Engvall (26), respectively.

In combination with primary mouse monoclonal antibodies we used biotinylated horse anti-mouse IgG (Vector Laboratories, Inc., Burlingame, CA) and DTAF (dichlorotriazinyl-amino-fluorescein)-conjugated streptavidin (Jackson ImmunoResearch, Inc., West Grove, PA) as secondary and tertiary steps. In combination with primary human antibodies we used mouse anti-human IgG (Southern Biotechnology Associates [SBA], Inc., Birmingham, AL) and FITC-conjugated goat anti-mouse IgG (Becton Dickinson, San Jose, CA). For double immunofluorescence with primary moabs of murine (1D1) and rat (439-9B) species we used goat anti-mouse IgG (SBA) and FITC-conjugated goat anti-rat IgG (SBA) and LRSC (lissamine rhodamine)-conjugated streptavidin (Jackson ImmunoResearch, Inc.) as secondary and tertiary steps.

Immunofluorescence procedures. 4- $\mu \mathrm{m}$ cryostat sections of skin specimens were processed for immunofluorescence as previously described (27). Fluorescence overlay antigen mapping was performed as recently described $(27,28)$. Digital video microscopic images of tissue sections were obtained with a newly developed imaging system with long exposure times designed for the detection of very low levels of fluorescence (29).

For immunofluorescence microscopy on cells, keratinocytes were resuspended and seeded onto coverslips in keratinocytes medium (DME-F12/HAM 2:1, 20\% FCS, $0.4 \mu \mathrm{g} / \mathrm{ml}$ hydrocortisone, $10 \mathrm{ng} / \mathrm{ml}$ EGF, $10 \mu \mathrm{g} / \mathrm{ml}$ non-essential amino acids and $10^{-10}$ choleratoxin) for 2-4 d. Cells were fixed with $1 \%$ formaldehyde in PBS for 10 min and permeabilized with $0.5 \%$ Triton $\mathrm{X}-100$ for $5 \mathrm{~min}$ at room temperature. After rinsing and blocking in 1\% BSA in PBS for 20 min, the permeabilized cells were incubated with primary antibody for $30 \mathrm{~min}$ at $37^{\circ} \mathrm{C}$. The cells were stained and washed as described for the tissues. The coverslips were viewed under a Nikon microscope extended with a confocal scanning laser microscope (MRC-600, Biorad, UK).

Electron microscopy. For electron microscopy routine procedures were followed as previously described (27).

Cell culture. From GABEB patient 1 a punch biopsy (diameter 6 $\mathrm{mm}$ ) of involved skin of the upper arm was taken. Epidermal cells were cultured according to a modified procedure of Rheinwald and Green (30). Briefly, epidermal cells were dissociated from the tissue specimen 
by a 30-min incubation with trypsin and grown in two $25-\mathrm{cm}^{2}$ culture flasks on a monolayer of lethally irradiated 3T3-mouse fibroblasts in keratinocytes medium (DME-F12/HAM 2:1, 20\% FCS, $0.4 \mu \mathrm{g} / \mathrm{ml}$ hydrocortisone, $10 \mathrm{ng} / \mathrm{ml}$ EGF, $10 \mu \mathrm{g} / \mathrm{ml}$ non-essential amino acids and $10^{-10}$ choleratoxin ).

Immunoblot procedure. At subconfluence cultured keratinocytes were lysed by overlaying them with demineralized water. The lysis liquid was discarded and the remaining cellular constituents solubilized with SDS-PAGE denaturation buffer $(10 \mathrm{mM}$ Tris- $\mathrm{HCl}, \mathrm{pH} 6.8$ containing $1 \mathrm{mM}$ EDTA, 2.5\% SDS, 5\% 2-mercaptoethanol, and $10 \%$ glycerol). Samples were heated for $10 \mathrm{~min}$ at $100^{\circ} \mathrm{C}$ and centrifuged at $14,000 \mathrm{~g}$ for $15 \mathrm{~min}$. The supernatant was collected and stored at $-80^{\circ} \mathrm{C}$ until used for immunoblot. Control cultures of normal keratinocytes from tissue obtained by breast reduction served as control. SDS-PAGE was performed according to Laemmli (31), using 5\% slab gels in the BioRad Mini Protean II electrophoresis apparatus (BioRad Laboratories, Richmond, CA). After separation the gel was blotted to nitrocellulose using the Mini Protean II blotting unit. After transfer the nitrocellulose was blocked with TTBS-buffer $(20 \mathrm{mM}$ Tris- $\mathrm{HCl}$ pH 7.5, $500 \mathrm{mM}$ $\mathrm{NaCl}, 0.05 \%$ Tween-20) and incubated with 1:300 diluted serum S3105 and 1:100 diluted monoclonal antibodies $1 \mathrm{~A} 8 \mathrm{c}$ and $5 \mathrm{E}$.

In combination with primary human antibodies we used affinity purified mouse anti-human IgG, Fc-fragment specific (Jackson ImmunoResearch, Inc.) and blotting grade affinity purified alkaline phosphatase conjugated goat anti-mouse IgG (BioRad), as secondary and tertiary steps. In combination with primary mouse antibodies we used goat anti-mouse IgG (Jackson ImmunoResearch, Inc.) followed by alkaline phosphatase conjugated rabbit anti-goat IgG (Jackson ImmunoResearch, Inc.).

Northern blot analysis. Total RNA was isolated from cultured keratinocytes of patient 1 , normal human keratinocytes and from the squamous cell carcinoma cell line UMSCC-22B using ULTRASPEC RNA reagent (Biotex lab., Inc., Houston, TX), separated on a $1 \%$ agarose gel containing $6 \%$ formaidehyde and transferred to nitrocellulose by a standard procedure (32). The nitrocellulose filter was hybridized for 16 $\mathrm{h}$ at $42^{\circ} \mathrm{C}$ with ${ }^{32} \mathrm{P}$-labeled cDNA probes in $50 \%$ formamide, $5 \times \mathrm{SSC}$, $50 \mathrm{mM} \mathrm{NaH} \mathrm{PO}_{4}, \mathrm{pH} 6.8,0.1 \%$ SDS, $100 \mu \mathrm{g}$ herring sperm DNA and $2 \times$ Denhardt's solution $(1 \times=0.02 \%$ polyvinylpyrrolidone, $0.02 \%$ Ficoll and $0.02 \%$ bovine serum albumin). The filters were washed two times with $2 \times \mathrm{SSC} / 0.1 \%$ SDS and once with $1 \times \mathrm{SSC} / 0.1 \%$ SDS at $42^{\circ} \mathrm{C}$, dried and exposed to Kodak X-OMAT AR film with intensifying screen at $-70^{\circ} \mathrm{C}$. The following cDNA probes were used: $(a)$ a $782-$ kb fragment, position 1771 to 2553 (4), coding for the extracellular part (NC16a en C15 domains) of BP180 and $(b)$ a 700-kb fragment coding for actin. The cDNA probes were ${ }^{32} \mathrm{P}$-labeled using Random primer labelling kit (GIBCO BRL, Gaithersburg, MD).

\section{Results}

Clinical description. The three GABEB patients had all the characteristics as summarized in the introduction (Fig. 1). The alopecia was not limited to terminal hair but also involved vellus hair. Mucous membranes were mildly affected: the patients occasionally had red eyes, nasal blood crusts, and oral erosions without dysphagia. Hypergranulation as in Herlitz disease was absent. The large naevocytic naevi as reported by Hintner and Wolf (15) in their GABEB family were not present in our patients.

GABEB patient 3 was unique. She was the only patient who had areas of skin with normal adherence and normal sensibility by touch. Examination by touching and rubbing disclosed a symmetrical patchy distribution of these areas over the extensor surface of the elbow, forearm, wrist, and hands (thenar and extensor surface of index and middle finger). We could not induce blisters after extensive rubbing in these areas. In contrast blisters could easily be evoked in similarly looking, clinically

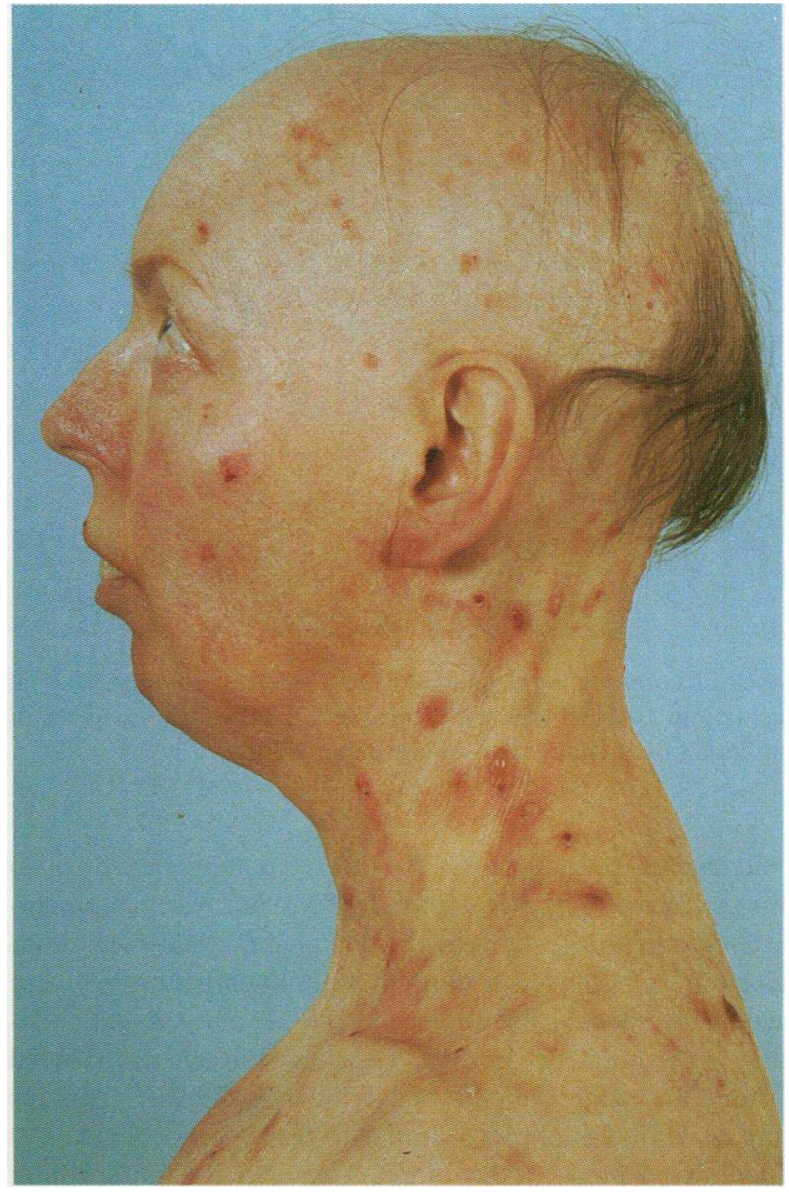

Figure 1. Clinical appearance of generalized atrophic benign epidermolysis bullosa. Typical scalp alopecia with follicular atrophy (patient 2).

normal but involved skin of the same patient. Patients 1 and 2 did not have any skin areas that were not-involved: all parts of the body were fragile.

Electron microscopy and immunofluorescence studies of skin biopsy material. Electron microscopy of affected skin of the GABEB patients revealed a split through the lamina lucida. In specimens from clinically normal and from lesional GABEB skin, hemidesmosomes were rudimentary and received less intermediate filaments than hemidesmomes from specimens from a healthy individual (Fig. 2). Despite the hypoplastic structure, some hemidesmosomes still possessed a subbasal dense plate.

Antigen mapping of the affected skin of the GABEB patients by immunofluorescence showed laminin-5 (GB3), laminin-1 and collagen type VII (LH7:2) exclusively in the blister floor and pan-keratin CK1 (Dako A/S, Glostrup, Denmark) exclusively in the blister roof. Immunofluorescence of clinically normal skin with monoclonal antibodies to laminin-5 and the $\alpha 6 \beta 4$ integrin gave normal results, as previously published for patient 1 (27).

The immunofluorescence results with skin tissues are summarized in Table II. There was no binding of moab 1D1 in the epidermal basement membrane zone (EBMZ) in the clinically normal, involved skin from the upper arm of the GABEB patients (Fig. $3 A$ ). In specimens from areas of not-involved skin of GABEB patient 3, unreduced staining with 1D1 occurred in patches along the EBMZ making up $\sim 50 \%$ of the total length 


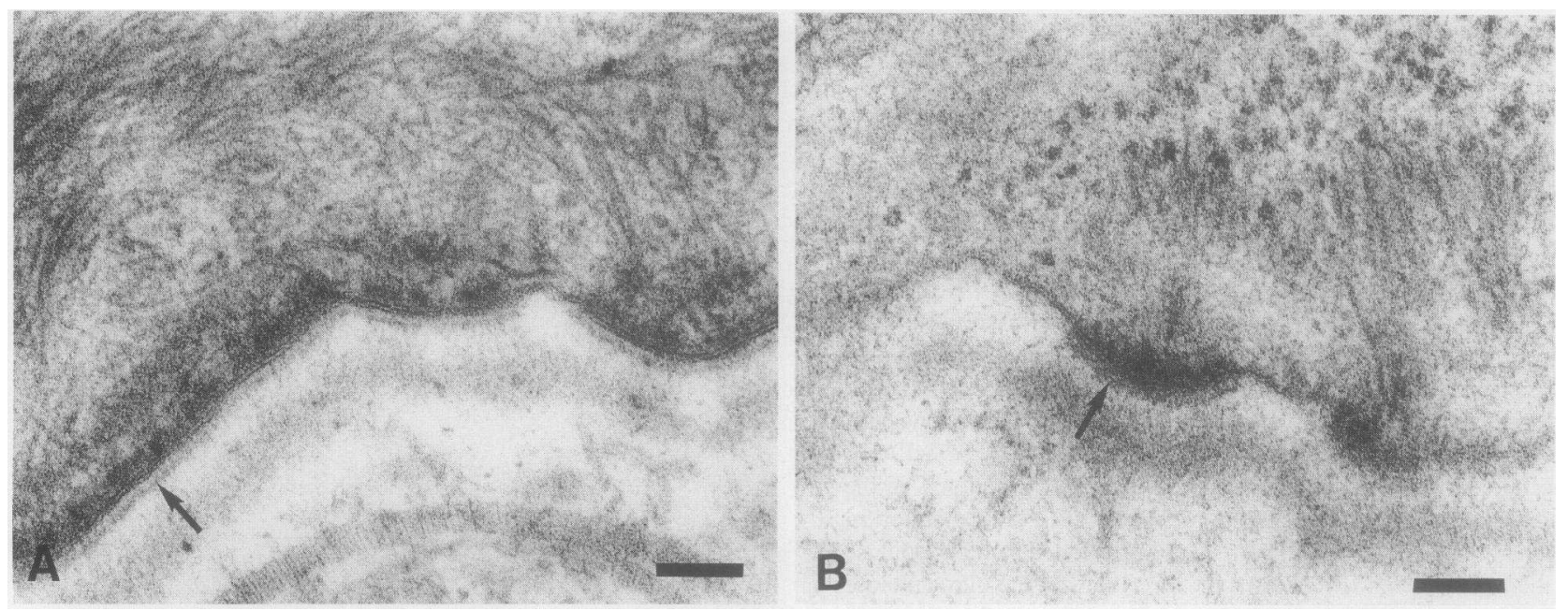

Figure 2. Electron micrographs of skin of a normal individual $(A)$ and of clinically normal skin of GABEB patient $1(B)$. Hemidesmosomes are rudimentary in GABEB $(B)$ and receive less intermediate filaments than normal hemidesmosomes $(A)$. Subbasal dense plates (arrows) are still present in GABEB hemidesmosomes. Bars, $100 \mathrm{~nm}$.

and interrupted by patches of $50-100 \mu \mathrm{m}$ long in which no staining occurred (Fig. $4 A$ ). Staining for other basement membrane components like integrin $\alpha 6 \beta 4$ (Fig. $4 B$ ), HD-1, laminin1 and laminin-5 showed a normal continuous pattern at these sites (data not shown). However, the $180-\mathrm{kD}$ antigen was undetectable in the clinically normal, involved skin and in involved skin from the same patient. In all the non-GABEB patients binding of 1D1 was normal, similar to controls.

Binding of moab 1A8c directed against the intracellular domain of BP180 did not occur in the specimens of the GABEB patients (Fig. 3B), nor was there reactivity with the polyclonal antibody R306.3 against an extracellular BP180 epitope (Fig. $3 C$ ). In specimens from not-involved skin of patient 3 the staining patterns of $1 \mathrm{~A} 8 \mathrm{c}$ and $\mathrm{R} 306.3$ were similarly interrupted as with moab $1 \mathrm{D} 1$.

Also binding of moab R815 to BP230 was normal in all GABEB specimens (Fig. $3 \mathrm{D}$ ). The other JEB specimens, stained also normally with antibodies to BP230, and with BPserum (S3105). The presence of BP230 in GABEB skin was confirmed with moabs $5 \mathrm{E}$ and $10 \mathrm{D}$ both producing an unreduced linear pattern. BP230 was consistently found in the blister roofs in GABEB specimens. Binding of moab HD-121 to $500-\mathrm{kD}$ HD1 plaque protein was normal in all JEB skin samples studied (data not shown).

We checked for variable BP antigen binding, as was found by Fine et al. (6) in the skin of patients with EB simplex using polyclonal BP-sera. However, in all six patients whom we studied, the reactivity with monoclonal antibodies 1D1 and R815 appeared to be normal.

Immunofluorescence and immunoblot studies of cultured keratinocytes. Immunofluorescence of cultured keratinocytes from patient 1 1D1 (Fig. 5 A), 1A8c and S2204 was negative. Nevertheless, the other hemidesmosomal components $500-\mathrm{kD}$ HD1, $\alpha 6$ and $\beta 4$ (Fig. $5 B$ ) were distributed in the normal pattern of dotted tracks which is characteristic for hemidesmosomal components.

To further confirm the absence of BP180, immunoblotting on extracts of cultured keratinocytes from GABEB patient 1

Table II. Epidermal Basement Membrane Zone Staining with Antibodies against Laminin-5, BP230 and HDI in Clinically Normal Skin of Patients with Junctional Epidermolysis Bullosa

\begin{tabular}{|c|c|c|c|c|c|c|c|c|c|}
\hline & & GB3 & 1D1 & $1 \mathrm{~A} 8 \mathrm{c}$ & R306.3 & S2204 & R815 & $5 \mathrm{E}$ & 10D \\
\hline Patient No. & Patient & laminin-5 & BP180ec & BP180ic & BP180ec & BP180 & $\mathrm{BP} 230$ & BP230 & BP230 \\
\hline 0 & Human controls & + & + & + & + & + & + & + & + \\
\hline 1 & GABEB & + & - & - & - & - & + & + & + \\
\hline 2 & GABEB & + & - & - & - & - & + & nd & nd \\
\hline 3 & GABEB & + & $-*$ & $-*$ & $-^{*}$ & $-*$ & + & nd & nd \\
\hline 4 & Lethal JEB & - & + & nd & nd & nd & + & nd & nd \\
\hline 5 & Lethal JEB & - & + & nd & nd & nd & + & nd & nd \\
\hline 6 & Lethal JEB & - & + & nd & nd & nd & + & nd & nd \\
\hline 7 & Lethal JEB & + & + & nd & nd & nd & + & nd & nd \\
\hline 8 & Cicatricial JEB & + & + & nd & nd & nd & + & nd & nd \\
\hline 9 & Pretibial JEB & + & + & nd & nd & nd & + & nd & nd \\
\hline
\end{tabular}

+ , Uniformly present; - , absent; and $n d$, not done. $*$ Absent in clinically normal, involved skin, focally present in not-involved skin areas. 

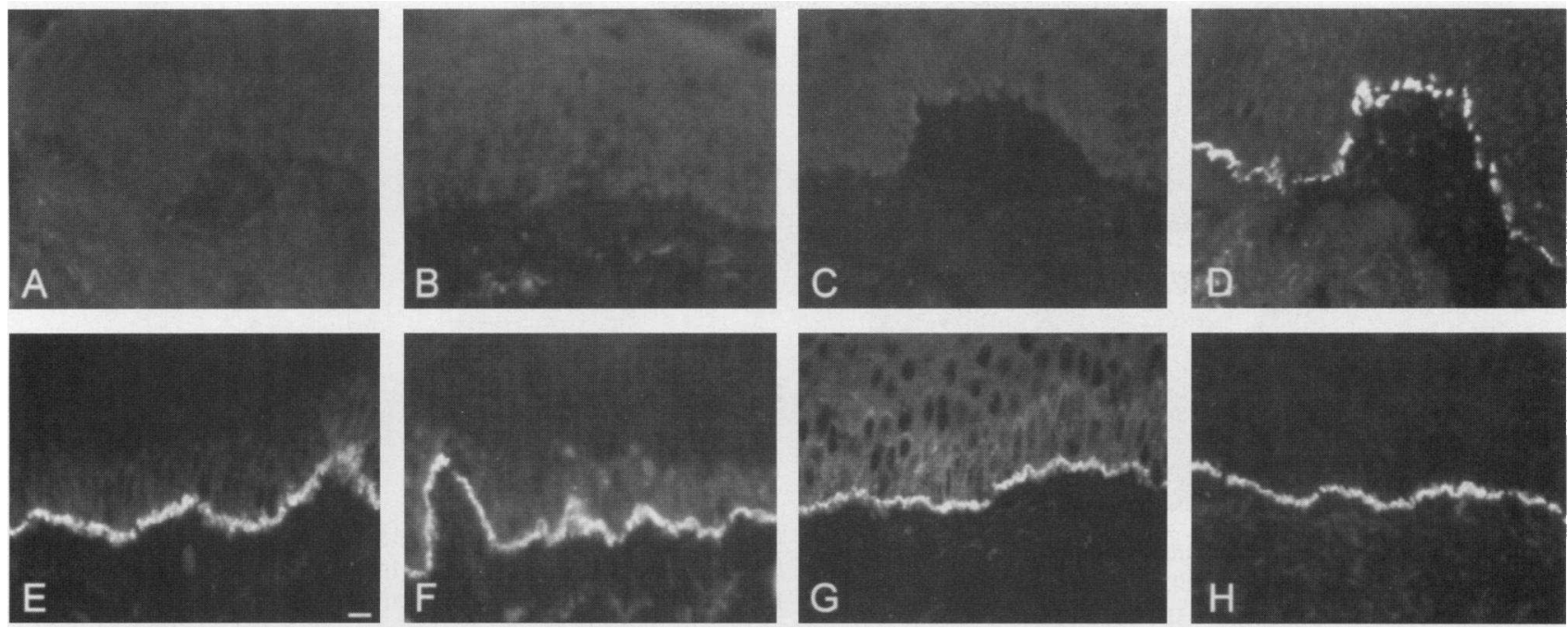

Figure 3. Immunofluorescence of clinically normal skin from GABEB patient $1(A-D)$ and of control skin $(E-H)$ using antibodies $1 D 1(A$ and $E$ ), $1 \mathrm{~A} 8 \mathrm{c}$ ( $B$ and $F$ ), and R306.3 ( $C$ and $G$ ) to BP180 and R815 ( $D$ and $H$ ) to BP230. Antibodies to BP180 did not react with GABEB skin $(A-$ $C$ ), whereas antibody to BP230 produced normal staining $(D)$. (Bar, $10 \mu \mathrm{m}$ ).

was performed. BP180 was not detected with monoclonal antibody $1 \mathrm{~A} 8 \mathrm{c}$ and the $180-\mathrm{kD}$ band was absent, when BP-serum S3105, which recognizes both BP230 and BP180 in control keratinocyte extracts was used (Fig. 6). The 230-kD bullous pemphigoid antigen was demonstrable by immunoblot in skinextracts of the GABEB patient. The presence of BP230 in GABEB keratinocytes was confirmed with moab 5E to BP230.

Northern blot analysis. Northern blot analysis of RNA isolated from cultured keratinocytes of patients 1 showed BP180 transcripts of 6.0 and $4.9 \mathrm{~kb}$ (Fig. $7 \mathrm{~A}$, lane 2). The size of these transcripts is not different from those in the squamous cell carcinoma cell line UMSCC-22B (Fig. $7 A$, lane $l$ ), and are consistent with the sizes of the transcripts reported for BP180 (4). In normal keratinocytes, only the 6.0-kb BP180 transcript was detected (Fig. $7 \mathrm{~B}$, lane $l$ ). The level of expression in normal keratinocytes and UMSCC-22B cells were comparable, whereas it was greatly reduced in the keratinocytes from patient 1 (Fig. $7 A$, lane 2 ). Control hybridizations with a probe for actin showed that equal amounts of RNA are present on the filters. Thus, the amount of BP180 mRNA was reduced in cultured keratinocytes of the GABEB patient.

\section{Discussion}

It is shown for the first time that the BP180 molecule is involved in the hereditary disorder: generalized atrophic benign epidermolysis bullosa (GABEB). In our previous study we found that the expression of the "bullous pemphigoid antigen" was not reduced in the GABEB patient 1 of the present study (27). In retrospect, however, we realized that we had used serum from a patient with bullous pemphigoid, which later as shown in the immunoblot appeared to contain in addition to antibodies to BP180, antibodies to the $230-\mathrm{kD}$ bullous pemphigoid antigen. Therefore, the deficiency of BP180 could not be detected by this serum. The use of monoclonal antibodies specific for the two distinct BP antigens has now revealed this deficiency.

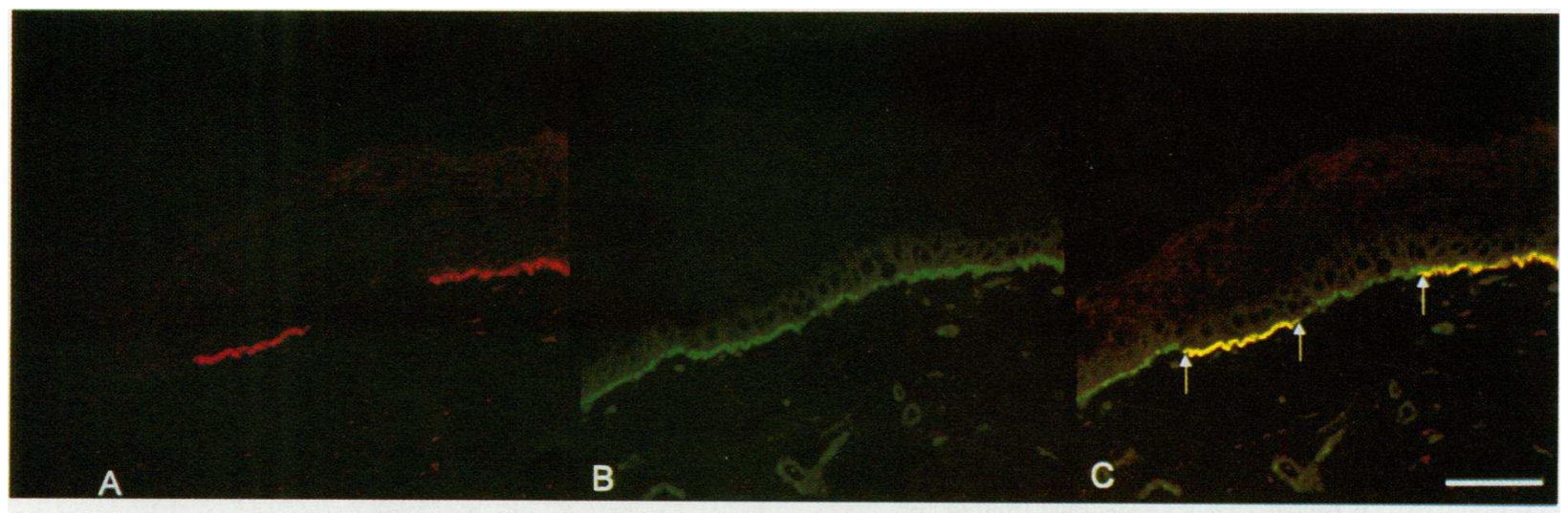

Figure 4. Fluorescence overlay antigen mapping $(C)$ of a skin section from clinically normal not-involved skin of GABEB patient 3 using moabs $1 \mathrm{D} 1$ to $\mathrm{BP} 180(A)$ and $439-9 \mathrm{~B}$ to the integrin $\beta 4$ subunit $(B)$. BP180 $(A)$ is interrupted along the epidermal basement membrane on stretches between $50-100 \mu \mathrm{m}$ long, while the $\beta 4$ subunit $(B)$ is continuously present in the same section. The overlay image $(C)$ of $A$ and $B$ shows yellow fluorescence on sites where both antigens are present (between arrows). The absence of BP180 cannot be attributed to artifact or sequelae of blistering. (Bar, $50 \mu \mathrm{m}$ ). 

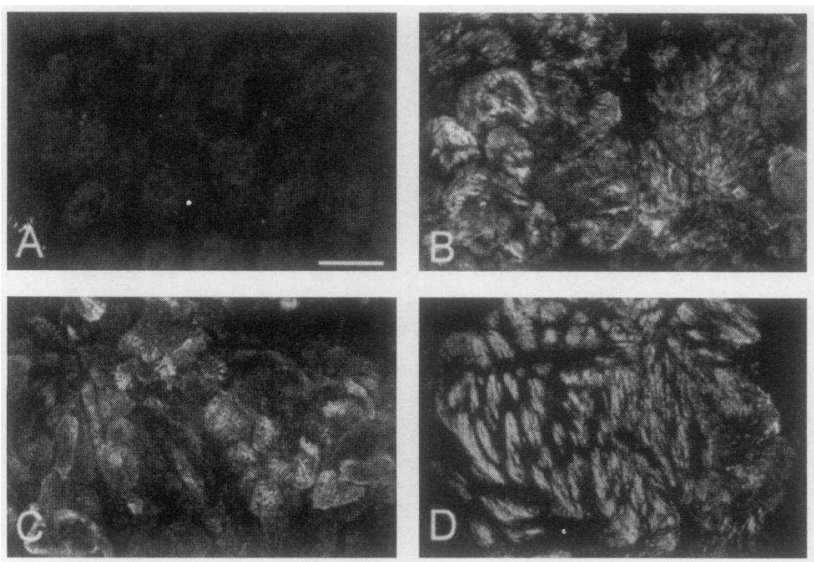

Figure 5. Immunofluorescence of cultured keratinocytes in vitro from GABEB patient $1(A$ and $B)$ and control $(C$ and $D)$ stained with moab $1 \mathrm{D} 1$ to BP180 ( $A$ and $C$ ) and moab 4.3E1 to integrin $\beta 4$ ( $B$ and $D$ ). GABEB keratinocytes do not express BP180, but staining for the integrin $\beta 4$ shows the normal pattern of dotted arrays representing stable anchoring complexes. (Bar, $25 \mu \mathrm{m}$ ).

The failure of antibody binding to BP180 in the GABEB patients is not restricted to one anatomical site since results with biopsies from alternative involved but clinically normal sites were identical. BP180 could also not be detected by immunofluorescence or immunoblot in keratinocytes cultured under standard conditions. However, in areas of not-involved, clinically normal skin from patient 3 , BP180 was interrupted along

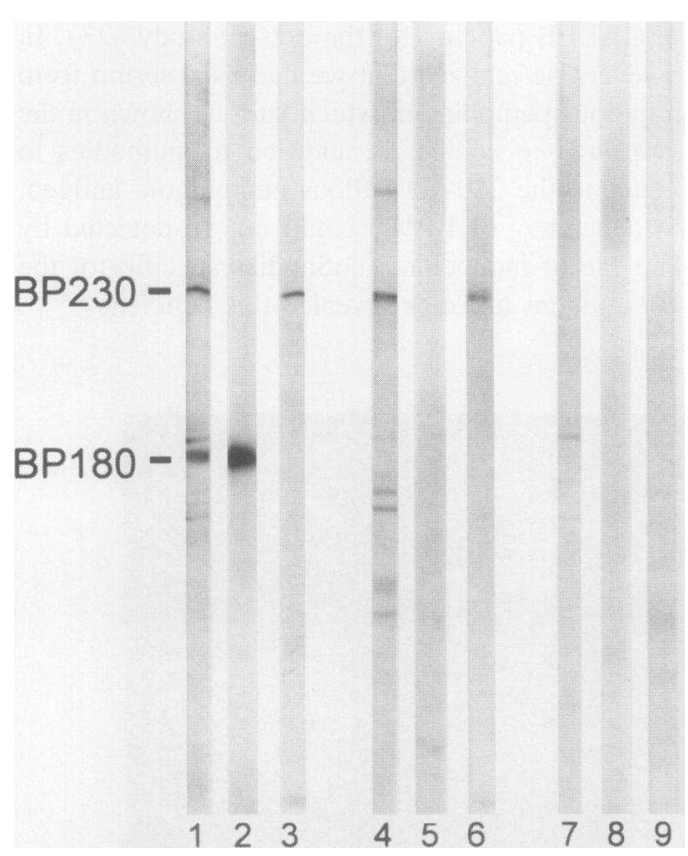

Figure 6. Immunoblot of extracts of cultured keratinocytes from control (lanes 1-3), from GABEB patient 1 (lanes 4-6), and from mouse fibroblasts (lanes 7-9) using BP-serum S3105 (lanes 1, 4, 7), moab 1A8c to BP180 (lanes 2, 5, 8) and moab 5E to BP230 (lanes 3,6, 9). Note that in the GABEB patient BP180 is missing (lanes 4,5) and BP230 is present (lanes 4,6). The mouse fibroblasts were included for control on irradiated $3 \mathrm{~T} 3$ cells used in keratinocyte cultures.

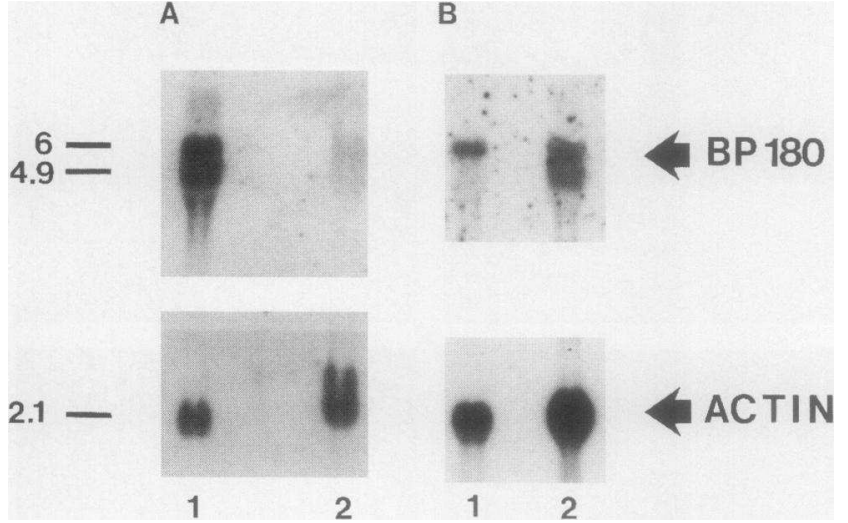

Figure 7. Northern blot analysis. (A) Total RNA ( $10 \mu \mathrm{g} /$ lane) from UMSCC-22B cells $(A$, lane 1$)$ and from cultured keratinocytes of GABEB patient $1(A$, lane 2$)$ was electrophoretically separated on $1 \%$ agarose/formaldehyde gel, transferred to nitrocellulose filter and probed with ${ }^{32} \mathrm{P}$-labeled cDNA coding for BP180 and actin. The BP180 mRNA signal is reduced, but not that of actin mRNA, in keratinocytes of the patient. ( $B$ ) Total RNA ( $10 \mu \mathrm{g} /$ lane) from normal human keratinocytes $(B$, lane 1$)$ and from UMSCC-22B cells ( $B$, lane 2 ) were assayed the same way as in $A$. The level of expression of BP180 mRNA in normal keratinocytes and UMSCC-22B cells were comparable.

$50 \%$ of the EBMZ, while other basement membrane components were not interrupted (Fig. 4).

The interrupted presence of BP180 in the not-involved skin of patient 3 is apparently sufficient to prevent skin blistering in these areas. This patient had an affected sibling, who is deceased and who therefore could not be studied. The BP180-positive patches might have been present only in patient 3 and might reflect somatic mosaicism in this patient: part of the cells escaped the generalized autosomal recessive disorder. This could be the result of somatic reversion due to recombination or to a reverse mutation of one of the two different mutations originally present on the two separate BP180 alleles (compound heterozygote), thus rendering this allele normal. If somatic reversion did happen in a putative primordial stem cell, then the BP180positive patches with a diameter of $50-100 \mu \mathrm{m}$ might reflect the area in which the keratinocytes, supplied by divisions of one stem cell, are localized.

The BP180 deficiency was exclusively found in the GABEB patients and not in the other JEB patients studied. We found no abnormal expression of the hemidesmosomal plaque proteins BP230 or HD1 in any of the JEB patients. In the three GB3deficient lethal JEB patients the disease is obviously the result of a defect in the laminin-5 genes (33). However, in the GB3positive lethal JEB patient (No. 7) and in the two non-lethal JEB patients (cicatricial JEB No. 8 and localized JEB No. 9) in this study, the molecular defect remains unknown.

The category generalized JEB mitis to which GABEB belongs, encompasses also nonlethal JEB patients with reduced laminin-5 (GB3) expression (34), in which recently a homozygous mutation in the LAMC2 gene have been demonstrated (35). Our GABEB patients did not have any reduction in the expression of laminin-5 (GB3). Thus the category generalized JEB mitis appears to be heterogeneous and GABEB appears to be a clinical subset characterized by alopecia. The question whether alopecia in generalized JEB mitis is characteristic for BP180 deficiency remains to be elucidated. 
The reduced amount of normal length BP180 mRNA in GABEB keratinocytes, as detected by Northern blot analysis, suggests a mutation in the BP180 gene resulting in reduced transcription or instability of the transcript. If that is the case, the lack of BP180 antigen in GABEB is due to reduced translation or to the presence of an early premature stop codon. An alternative hypothesis, namely that a post-translational failure of protein processing might explain the deficiency of BP180, is less likely because of the results of the Northern blot analysis. Moreover, the intracellular and extracellular epitopes of BP180 were undetectable, which would be unlikely if improper posttranslational protein folding had caused the deficiency. All together the evidence supports the total absence of the BP180 antigen in skin of GABEB patients.

As to the mode of inheritance, the pedigrees of our patients indicate a recessive form of the abnormal allele. Human BP180 has been cloned and sequenced. BP180 has interrupted collagenous domains in the extracellular region, which have determined some authors to classify the molecule as type XVII collagen (36). The gene is located on chromosome 10q24.3 (37). Molecular genetic studies are now in progress to further localize and identify the specific mutation responsible for the abnormality.

From this study it appears that the transmembrane molecule BP180 is essential for cell adhesion, since the deficiency of this molecule is associated with serious skin abnormalities. Autoimmunity against the extracellular domain of this molecule (20), as it occurs in bullous pemphigoid might also lead to detachment and blistering of skin. BP180 also appears to be critical for hair root anchorage. GABEB patients loose their terminal hair in childhood between the age of 5 and 12. The almost complete absence of the vellus hair should also be mentioned. Patients never develop a secondary hair pattern in puberty, although the gonads appear to function normally. It remains to be elucidated why the mucous membranes in patients with GABEB are involved to a lesser extent than skin, since BP180 is normally also present along the basement membranes of the cornea, the mouth, the ear, the nose, the throat, the esophagus and in the urogenital tract. It is possible that the kidney failure of patient 1 is the result of lesions in the urinary tract. Renal dysfunction has been associated with GABEB (38).

Knowing that BP180 is deficient in GABEB, the ultrastructure of the hemidesmosomes becomes significant. Notably, rudimentary hemidesmosomes were present in GABEB skin, some even possessed a subbasal dense plate (Fig. $2 B$ ). Thus, for hemidesmosomes to mature BP180 seems to be necessary, but it appears not to be critical for their initial assembly. In a previous study we found no abnormality of the expression of integrin $\alpha 6 \beta 4$ in GABEB (27). These findings emphasize the hypothesis that integrin $\alpha 6 \beta 4$ (and not BP180) plays a critical role in initial hemidesmosome assembly (39).

In conclusion, in this study we found that the BP180 antigen is deficient and the BP180 mRNA is reduced in GABEB, suggesting that BP180 gene is candidate gene for this hereditary blistering disorder.

\section{Acknowledgments}

We wish to thank Janny Scholma and Anna Tomson for culturing keratinocytes of the patient (University of Groningen, the Netherlands). We thank Johan Toonstra for referring patient 2 (University of Utrecht, The Netherlands). We gratefully acknowledge the gift of RNA from normal keratinocytes by Liesbeth van de Raaij-Helmer (Free University, Amsterdam, The Netherlands).

\section{References}

1. Tidman, M. J., and R. A. J. Eady. 1986. Hemidesmosome heterogeneity in junctional epidermolysis bullosa revealed by morphometric analysis. J. Invest. Dermatol. 86:51-56.

2. Ishiko, A., H. Shimizu, A. Kikuchi, T. Ebihara, T. Hashimoto, and T. Nishikawa. 1993. Human autoantibodies against the $230-\mathrm{kD}$ bullous pemphigoid antigen (BPAG1) bind only to the intracellular domain of the hemidesmosome, whereas those against the $180-\mathrm{kD}$ bullous pemphigoid antigen (BPAG2) bind along the plasma membrane of the hemidesmosome in normal human and swine skin. J. Clin. Invest. 91:1608-1615.

3. Nishizawa, Y., J. Uematsu, and K. Owaribe. 1993. HD4, a $180 \mathrm{kDa}$ bullous pemphigoid antigen, is a major transmembrane glycoprotein of the hemidesmosome. J. Biochem. (Tokyo). 113:493-501.

4. Giudice, G. J., D. J. Emery, and L. A. Diaz. 1992. Cloning and primary structural analysis of the bullous pemphigoid autoantigen BP180. J. Invest. Dermatol. 99:243-250.

5. Amagai, M., G. W. Elgart, V. Klaus-Kovtun, and J. R. Stanley. 1991. Southern analysis of the $230-\mathrm{kD}$ bullous pemphigoid antigen gene in normal humans, animals, and patients with junctional epidermolysis bullosa. J. Invest. Dermatol. 97:249-253.

6. Fine, J. 1985. Epidermolysis bullosa: variability of expression of cicatricial pemphigoid, bullous pemphigoid, and epidermolysis bullosa acquisita antigens in clinically uninvolved skin. J. Invest. Dermatol. 85:47-49.

7. Paller, A. S., J.-D. Fine, S. Kaplan, and R. W. Pearson. 1986. The generalized atrophic benign form of junctional epidermolysis bullosa. Arch. Dermatol. 122:704-710.

8. Heagerty, A. H. M., A. R. Kennedy, and R. A. J. Eady. 1986. Lethal junctional epidermolysis bullosa: a disorder of hemidesmosome formation but not bullous pemphigoid antigen? J. Invest. Dermatol. 87:144. (Abstr.)

9. Bruckner-Tuderman, L., S. Rüegger, B. Odermatt, Y. Mitsuhashi, and U. W. Schnyder. 1988. Lack of type VII collagen in unaffected skin of patients with severe recessive dystrophic epidermolysis bullosa. Dermatologica. 176:5764.

10. Labib, R. S., G. J. Anhalt, G. J., H. P. Patel, D. F. Mutasim, and L. A. Diaz. 1986. Molecular heterogeneity of the bullous pemphigoid antigens as detected by immunoblotting. J. Immunol. 136:1231-1235.

11. Owaribe, K., Y. Nishizawa, and W. W. Franke. 1991. Isolation and characterization of hemidesmosomes from bovine corneal epithelial cells. Exp. Cell Res. 192:622-630.

12. Hashimoto, T., M. Amaigai, T. Ebihara, S. Gamou, N. Shimizu, T. Tsubata, A. Hasegawa, K. Miki, and T. Nishikawa. 1993. Further analyses of epitopes for human monoclonal anti-basement membrane zone antibodies produced by stable human hybridoma cell line constructed with Epstein-Barr virus transformants. $J$. Invest. Dermatol. 100:310-315.

13. Hieda, Y., Y. Nishizawa, J. Uematsu, and K. Owaribe. 1992. Identification of a new hemidesmosomal protein, $\mathrm{HDl}$ : a major, high molecular mass component of isolated hemidesmosomes. J. Cell Biol. 116:1497-1506.

14. Fine, J.-D., E. A. Bauer, R. A. Briggaman, D. M. Carter, R. A. J. Eady, N. B. Esterly, K. A. Holbrook, S. Hurwitz, L. Johnson, and A. Lin. 1991. Revised clinical and laboratory criteria for subtypes of inherited epidermolysis bullosa. $J$. Am. Acad. Dermatol. 24:119-135.

15. Hintner, H., and K. Wolff. 1982. Generalized atrophic benign epidermolysis bullosa. Arch. Dermatol. 118:375-384.

16. Gedde-Dahl, T., Jr., and I. Anton-Lampecht. 1983. Epidermolysis bullosa. In Principles and Practice of Medical Genetics. A. E. H. Emory and D. L. Rimoin, editors. Churgchill-Livingstone Inc., New York. 672-687.

17. Tidman M. J., R. A. J. Eady, and R. A. Marsden. 1985. Non-lethal junctional epidermolysis bullosa. Br. J. Dermatol. 29(suppl):83.

18. Hashimoto, I., U. W. Schnyder, and I. Anton-Lamprecht. 1976. Epidermolysis bullosa hereditaria with junctional blistering in an adult. Dermatologica. 152:72-86.

19. Jonkman, M. F., M. C. J. M. de Jong, and J. B. van der Meer. 1994. Cicatricial junctional epidermolysis bullosa. J. Amer. Acad. Dermatol. 31:286288.

20. Giudice, G. J., D. J. Emery, B. D. Zelickson, G. J. Anhalt, Z. Liu, and L. A. Diaz. 1993. Bullous pemphigoid and herpes gestationis autoantibodies recognize a common non-collagenous site on the BP180 ectodomain. J. Immunol. 151:57425750 .

21. Verrando, P., B. Hsi, C. G. Yeh, A. Pisani, N. Serieys, and J.-P. Ortonne. 1987. Monoclonal antibody GB3, a new probe for the study of human basement membranes and hemidesmosomes. Exp. Cell Res. 170:116-128.

22. Leigh, I. M., R. A. J. Eady, A. H. M. Heagerty, P. E. Purkis, P. A Whitehead, and R. E. Burgeson. 1988. Type VII collagen is a normal component of epidermal basement membrane, which shows altered expression in recessive dystrophic epidermolysis bullosa. J. Invest. Dermatol. 90:639-642. 
23. Sonnenberg, A., H. Jansen, F. Hogervorst, J. Calafat, and J. Hilgers. 1987. A complex of platelet glycoproteins Ic and IIa identified by a rat monoclonal antibody. J. Biol. Chem. 262:10376-10383.

24. Hogervorst, F., I. Kuikman, E. Noteboom, and A. Sonnenberg. 1993. The role of phosphorylation in activation of the $\alpha 6^{\mathrm{A}} \beta 1$ laminin receptor. J. Biol. Chem. 18427-18430.

25. Kennel, S. J., L. J. Foote, R. Falcioni, A. Sonnenberg, C. D. Stringer, C Crouse, and M. E. Hemler. 1989. Analysis of the tumor-associated antigen TSP180. Identity with $\alpha 6 \beta 4$ in the integrin superfamily. J. Biol. Chem. 264:1551515521.

26. Hessle, H., L. Y. Sakai, D. W. Hollister, R. E. Burgeson, and E. Engvall. 1984. Basement membrane diversity detected by monoclonal antibodies. Differen tiation. 26:49-54.

27. Jonkman, M. F., M. J. C. M. de Jong, K. Heeres, and A. Sonnenberg. 1992. Expression of integrin $\alpha 6 \beta 4$ in junctional epidermolysis bullosa. J. Invest. Dermatol. 99:489-496.

28. Bruins, S., M. J. C. M. de Jong, K. Heeres, M. H. F. Wilkinson, M. F. Jonkman, and J. B. van der Meer. 1994. Fluorescence overlay antigen mapping of the epidermal basement membrane zone: I geometric errors. J. Histochem. Cytochem. 42:555-560.

29. Wilkinson, M. H. F., G. J. Jansen, and D. van der Waaij. 1992. Very low level fluorescence detection and imaging using a long exposure charge coupled device system. In Biotechnology Applications of Microinjection, Microscopic Imaging and Fluorescence. P. H. Bach, C. H. Reynolds, J. M. Clark, P. L. Poole, and J. Mottley, editors. Pergamon Press, London. 220-230.

30. Tomson, A. M., J. Scholma, E. H. Blaauw, H. J. Rosingh, and F. G. Dikkers. 1994. Improved in vitro generation of epithelial grafts with oral mucoa. Transplantation. 58:1282-1284.
31. Laemmli, U. K. Cleavage of structural proteins during assembly of the head of bacteriophage T4. 1970. Nature (Lond.). 227:680-685.

32. Sambrook, J., E. F. Fritsch, and T. Maniatis. 1989. Molecular Cloning: A Laboratory Manual. Cold Spring Harbor Laboratory Press, Cold Spring Harbor NY.

33. Aberdam, D., M. Galliano, J. Vailly, L. Pulkkinen, J. M. Bonifas, A. M Christiano, K. Tryggvason, J. Uitto, E. H. J. Epstein, J. Ortonne, and G. Meneguzzi. 1994. Herlitz' junctional epidermolysis bullosa is linked to mutations in the gene (LAMC2) for the $\gamma 2$ subunit of nicein/kalinin (LAMININ-5). Nat. Genet. 6:299-304.

34. Verrando, P., O. M. V. Schofield, A. Ishida-Yamamoto, D. Aberdam, O. Partouche, R. A. J. Eady, and J. Ortonne. 1993. Nicein (BM-600) in junctional epidermolysis bullosa: polyclonal antibodies provide new clues for pathogenic role. J. Invest. Dermatol. 101:738-743.

35. Pulkkinen, L., A. M. Christiano, T. Airenne, H. Haakana, K. Tryggvason, and J. Uitto. 1994. Mutations in the $\gamma 2$ chain gene (LAMC2) of kalinin/laminin 5 in the junctional forms of epidermolysis bullosa. Nat. Genet. 6:293-298.

36. Li, K., K. Tamai, M. L. Tan, and J. Uitto. 1993. Cloning of type XVII collagen. J. Biol. Chem. 268:8825-8834.

37. Li, K., D. Sawamura, G. J. Guidice, L. A. Diaz, M.-G. Mattei, M.-L. Chu, and J. Uitto. 1991. Genomic organization of collagenous domains and chromosomal assignment of human $180 \mathrm{kD}$ bullous pemphigoid antigen (BPAG2), novel collagen of stratified squamous epithelium. J. Biol. Chem. 266:2406424069.

38. Yamada, Y., S. Dekio, J. Jidoi, T. Ishimoto, and T. Yoshioka. 1990 Epidermolysis bullosa atrophicans generalisata mitis - report of a case with renal dysfunction. J. Dermatol. 17:690-695.

39. Jones, J. C. R., M. A. Kurpakus, H. M. Cooper, and V. Quaranta. 1991. A function for the integrin $\alpha 6 \beta 4$ in the hemidesmosome. Cell Regul. 2:427-438. 